\title{
Método de análise e dimensionamento do sistema fotovoltaico ongrid
}

A procura pela multiplicidade da fonte energética brasileira, tem se tornado cada vez mais essencial por conta de dois principais fatores. Primeiramente pelo atual cenário energético, em que, com a diminuição das chuvas e conseguintemente redução da energia gerada por hidrelétricas, aumentou-se, significativamente, o preço da energia. E ainda, pela necessidade de explorar recursos renováveis que trazem flexibilidade e sustentabilidade na sua utilização, isso faz com que a energia fotovoltaica seja uma opção para a sociedade. Diante deste cenário, as planilhas têm sido presentes nos projetos de sistema solar como um recurso ágil e prático de análise e dimensionamento dessas estruturas. A pesquisa consistiu em desenvolver uma ferramenta de dimensionamento e análise da viabilidade para projetos fotovoltaicos ongrid através de planilhas, relacionando métodos utilizados para dimensionamento das placas solares, produzindo uma ferramenta para dimensionamento de sistemas fotovoltaicos e identificando o ponto ótimo de investimento baseado no prazo de retorno.

Palavras-chave: Dimensionamento fotovoltaico; Planilha de dimensionamento solar; Energia Solar; Sistema fotovoltaico.

\section{Analysis and dimensioning method of the ongrid photovoltaic system}

The search for the multiplicity of the Brazilian energy source has become increasingly essential due to two main factors. Firstly due to the current energy scenario, in which, with the decrease of rainfall and the consequent reduction of the energy generated by hydroelectric plants, the price of energy increased significantly. And yet, by the need to explore renewable resources that bring flexibility and sustainability in their use, this makes photovoltaic energy an option for society. Given this scenario, spreadsheets have been present in solar system projects as an agile and practical resource for analysis and sizing of these structures. The research consisted of developing a sizing and feasibility analysis tool for ongrid photovoltaic projects through spreadsheets, relating methods used for sizing solar panels, producing a tool for sizing photovoltaic systems and identifying the optimal point of return-based investment.

Keywords: Photovoltaic sizing; Solar sizing worksheet; Solar energy; Photovoltaic system.

Topic: Engenharia Civil

Reviewed anonymously in the process of blind peer.
Received: 16/05/2019

Approved: 20/08/2019
Fernanda Santos de Carvalho iD

Instituto Tocantinense Presidente Antonio Carlos, Brasil

http://lattes.cnpq.br/1373218368904414

http://orcid.org/0000-0001-5107-709X

f.santos2016@hotmail.com

Eduardo Gouveia Santiago Lage

Instituto Tocantinense Presidente Antônio Carlos, Brasil

http://lattes.cnpq.br/4344949197847151

http://orcid.org/0000-0003-0011-2451

eduardogslage@gmail.com
Referencing this:

CARVALHO, F. S.; LAGE, E. G. S.. Método de análise e dimensionamento do sistema fotovoltaico ongrid. Technology Science, v.1, n.2, p.24-36, 2019. DOI:

http://doi.org/10.6008/CBPC2674-6425.2019.002.0004 


\section{INTRODUÇÃO}

A energia elétrica é uma das maiores conquistas da humanidade, pelas suas infindáveis utilidades. Segundo a agência nacional de energia elétrica (ANEEL), a forma de geração mais utilizada ao longo dos anos são as hidroelétricas, representou $88 \%$ em 2008, porém a água, é findável, por isso novos recursos infindáveis foram estudados para continuar gerando essa energia para as comunidades.

E um desses novos meios para gerar energia, é o sistema fotovoltaico. Existe agora um crescente interesse na utilização direta da energia solar para diversos fins. Buscando novas tecnologias para o uso de energias renováveis, o sistema fotovoltaico se encontra em crescente utilização. Com isso, tem-se explorado novos materiais para o avanço da tecnologia fotovoltaica.

A energia solar fotovoltaica é definida como a energia gerada através da conversão direta da radiação solar em eletricidade. Isto se dá, por meio de um dispositivo conhecido como célula fotovoltaica que atua utilizando o princípio do efeito fotoelétrico ou fotovoltaico. Portanto, seguindo o princípio de sustentabilidade e agilidade, este trabalho consiste em produzir uma ferramenta para dimensionar sistema fotovoltaico, objetivando analisar a sua eficiência para viabilizar a instalações de todos os tipos de unidades.

\section{REVISÃO TEÓRICA}

\section{Sistemas Fotovoltaicos}

A energia solar fotovoltaica é vista como a energia produzida por meio da conversão da radiação solar em eletricidade. Por meio de um dispositivo conhecido como célula fotovoltaica que é feita essa conversão, que atua com o princípio do efeito fotoelétrico ou fotovoltaico (IMHOFF, 2007). A procura de novas tecnologias para o uso de energias renováveis, os sistemas fotovoltaicos têm crescido no mercado. Com isso, tem-se estudado novos materiais e realizado pesquisas para o avanço da tecnologia fotovoltaica. (CEMIG, 2012).

Portanto, é um sistema de energia projetado para fornecer energia solar por meio da tecnologia fotovoltaica, a maioria dos sistemas no mercado é diretamente ligada à rede (ongrid). E para fins de análise e cálculo de dimensionamento desses sistemas, foram conhecidos seus componentes, incluindo os painéis solares, inversores, cabeamento, montagem, e outros acessórios para esse sistema.

\section{Sistemas Ligados à Rede (Ongrid)}

Os sistemas ongrid são aqueles que trabalham juntamente à rede elétrica da distribuidora de energia. De maneira geral, o painel fotovoltaico gera energia elétrica em corrente contínua e, converte-a para corrente alternada, e é inserida na rede de energia elétrica. Essa conversão é feita pela utilização de inversores, que realiza a interface entre o painel e a rede elétrica.

\section{Painéis Solares}

O painel solar é o principal componente do sistema fotovoltaico de produção de energia. Eles são 
formados por células fotovoltaicas associadas, eletricamente, em serie e/ou paralelo, conforme as tensões e/ou corrente definidas em projeto. A junção destes painéis é chamada de gerador fotovoltaico e constituem a parte responsável no processo de adaptação da irradiação solar e a sua transformação em energia elétrica.

\section{Inversores}

Os inversores são dispositivos eletrônicos que distribuem a energia elétrica em corrente alternada, proveniente de uma fonte de energia elétrica em corrente contínua.

\section{Corrente Elétrica}

É o curso de cargas elétricas portando pequenas partículas chamadas elétrons. A corrente contínua (CC) é descrita como o fluxo contínuo de elétrons em uma mesma direção, com variação baixa na força da corrente, já a corrente alternada (CA) é o fluxo descontínuo de elétrons e variação de direção é chamado de corrente alternada, que são usadas nos eletrodomésticos.

\section{Posicionamentos das Placas}

O posicionamento das placas é importante para um melhor aproveitamento das incidências solares. Respeitando as coordenadas geográficas e o limite de sombras de prováveis objetos. Para instalar as placas é preciso uma área mínima de telhado, na empresa ou residência. E que a orientação dos sistemas é importante por estarmos no hemisfério sul do planeta, e as placas precisam ser instaladas de 10 até 26 graus de inclinação ao Norte.

\section{Tracker (Seguidor Solar)}

O tracker é um sistema de monitoramento solar que inclina o painel durante o dia de acordo com o posicionamento do sol, ou a área mais brilhante no caso de dias nublados (SOLIENS, 2017).

\section{Células fotovoltaicas}

Células solares ou fotovoltaicas são elementos responsáveis pela modificação da energia solar em energia elétrica. Estas usam as propriedades dos materiais semicondutores (na maior parte, o Silício). 0 processo de geração de energia em corrente contínua é chamado de Efeito Fotoelétrico. Cada célula solar gera cerca de 0,4 volts no seu ponto de máxima potência, portanto é necessário conectá-las de forma devida para as tensões desejadas (FIGUEIRA, 2014).

\section{Custos da Energia Elétrica}

A Aneel é a agência que regula o setor elétrico brasileiro é a agência com finalidade de produção, transmissão e distribuição de energia elétrica. É através desse site que temos respaldo para retratar os custos energéticos das unidades 'consumidoras. O custo da energia se dá pela somatória de encargos, impostos e custos de produção e distribuição. Segundo a ANEEL (1996) a tarifa residencial de maior valor no ranking da 
região norte está na Amazônia, e em quarto lugar está o Tocantins, com valor de 0,605R\$/kWh, sem contemplar tributos e elementos que fazem parte da conta de luz.

Quanto maior a taxa tarifária, mais viável é instalar as placas solares, e quanto mais altas os valores das tarifas, menos tempo leva para o investimento ser pago. Segundo a Energisa (1905), o cálculo do valor da conta é com base no consumo mensal registrado, logo após é adicionado os tributos em vigor mais a CIP (contribuição de iluminação pública) do município.

\section{ICMS}

Segundo Cabral (2016) o ICMS é o Imposto sobre Operações relacionadas à Circulação de Mercadorias e Prestação de Serviços de transporte interestadual e intermunicipal e de comunicação.

\section{PIS/PASEP E COFINS}

Os recursos do PIS são designados ao pagamento do seguro-desemprego, abono e participação na receita dos órgãos e para os trabalhadores públicos e privados, onde o PIS é indicado aos funcionários de empresas privadas, administrado pela Caixa Econômica Federal, e o PASEP destinado aos servidores públicos, administrado pelo Banco do Brasil. E os recursos da COFINS são determinados principalmente para a área da saúde.

\section{Taxa de Iluminação Pública}

Energisa (1905) diz que os municípios são encarregados por cobrar da população pelos recursos necessários para o custeio dos serviços de iluminação pública. A CIP é um tributo definido no art. 149-A da Constituição Federal de 1988 e a instituição da contribuição e seus relativos valores são estabelecidos para cada localidade através de Leis e Decretos Municipais.

\section{Bandeira Tarifária}

Para a Aneel (1996), as tarifas apresentam modalidades diferentes. Bandeira Verde significa que a situação está favorável e não sofrem acréscimos, a bandeira amarela diz que são condições menos favoráveis, sofrendo o acréscimo de $\mathrm{R} \$ 0.010 \mathrm{kWh}$, já a bandeira vermelha as condições de geração de energia sofrem maiores acréscimos, e segue por dois patamares, no primeiro patamar a tarifa aumenta R\$ $0,030 \mathrm{kWh}$, no segundo patamar $\mathrm{R} \$ 0,50 \mathrm{kWh}$. As bandeiras tarifárias são para ser utilizadas quanto ocorre alguma mudança que aumente o custo da energia produzida no país, como falta de água nos reservatórios, que faz a necessidade de ligar usinas termoelétricas que tem custos altos.

\section{METODOLOGIA}

Pesquisa de caráter descritivo, com apresentação de análises quantitativas da energia fotovoltaica. As fontes de pesquisa foram livros, softwares, normas técnicas, análise de tarifas da energia elétrica, internet e planilhas elaboradas através do Microsoft Office Excel, analisando e dimensionando de forma eficaz os 
sistemas fotovoltaicos.

\section{Metodologia de Coleta dos Elementos do Banco de Dados}

Para formar um banco de dados sobre o tema, optou-se por fazer uma revisão bibliográfica nos diversos sites com pesquisa e trabalhos nessa área, assim foram coletados os métodos de cálculo para instalação das placas solares.

\section{Metodologia de Cálculo}

Para que os cálculos sejam executados nas planilhas que foram elaboradas como foco principal do trabalho, foi necessário informar alguns dados e equações como consumo médio, custo mínimo, equações de dimensionamento fotovoltaico, e as discrições dos valores somados no cálculo da fatura de energia elétrica, encontradas no site da Energisa e os demais em sites relacionados aos sistemas fotovoltaicos.

\section{Consumo Médio}

Para início do projeto foi pesquisado o consumo médio do cliente, fornecido na conta de energia, ou seu consumo mensal durante os últimos doze meses para a média, onde as produções de todos os meses são somadas e divididas por doze.

\section{Vistoria no Local da Implantação das Placas}

A vistoria do local onde o consumidor irá instalar as placas é necessária, pois o posicionamento dos módulos influencia no rendimento do sistema, para saber se pode existir sombreamento no local, qual a inclinação do telhado, entre outros fatores.

\section{Custo mínimo}

Sendo um sistema ongrid, ligados à rede, portanto houve um custo mensal da concessionária local a Energisa, para mantê-la em funcionamento. Essas ligações normalmente são divididas em três: Monofásico=conexão de $127 \mathrm{~V}, 220 \mathrm{~V}$ e $254 \mathrm{~V}$, custo de 30kWh mensais; Bifásico=comum em residências, conexão de $220 \mathrm{~V}$ e $380 \mathrm{~V}$, custo de 50kWh mensais; Trifásico=conexão de $220 \mathrm{~V}$ e $380 \mathrm{~V}$, custo de $100 \mathrm{kWh}$ mensais; Além desse custo da distribuidora, o custo de iluminação pública (CIP) é adicionado, entendendo que mesmo o sistema esteja gerando $100 \%$ da energia consumida, não poderá zerar a conta de energia.

\section{Cálculo do Dimensionamento de Sistemas Fotovoltaicos}

\section{Cálculo da Potência}

A saída de energia é a potência do aparelho multiplicado pelo seu tempo de uso, expresso na conta de luz como o consumo.

$$
E=P x T
$$


Na energia solar temos a equação sendo aplicada como:

$$
E g=\operatorname{Ptp} \times T e
$$

Eg- Energia de geração Ptp- Potência total dos painéis Te-Tempo de exposição ao sol

Assim pode-se isolar a potência para cálculo da quantidade de placas necessárias:

$$
P t p=\frac{\text { Eg }}{\mathrm{Te}}
$$

Porém o sistema de energia solar não é isento de perda de energia, podendo ter perdas pelos inversores, cabos e painéis, por isso será adicionado outro termo a equação:

$$
P t p=\frac{\mathrm{Eg}}{\mathrm{Te} \times \mathrm{Nr}}
$$

Nr-Rendimento

Eg-Energia de geração Ptp- Potência total dos painéis Te-Tempo de exposição ao sol

\section{Cálculo da Energia de Geração}

O dimensionamento da energia de geração é para ter menor custo de investimento e reduzir ao máximo a conta de energia, para isso fez-se o seguinte cálculo:

$$
\mathrm{Ec}=\mathrm{Cm}-\mathrm{L}
$$

Ec- Energia de geração (kWh/mês) $\mathrm{Cm}$-Consumo médio L-Ligação (bifásica)

Calcula-se esse valor em dias, pois a produção média diária deve ser superior a 6,67kWh durante o ano.

$$
E c d=\frac{\mathrm{Dg}}{30}
$$

Ecd=Energia de geração por dia $\mathrm{Dg}=$ Energia de geração

\section{Potência-Pico}

O tempo de exposição foi encontrado através dos dados solarimétricos da região de Porto Nacional/TO. Por meio do programa SunDate, que é ofertado pela CRESCESB (Centro de referência para as energias solar e eólica Sergio de S. Brito), pode ser encontrado a irradiação média mensal em qualquer ponto do território nacional. No site da CRESESB está no plano horizontal o HSP (horas de sol pico) de cada mês no município, e será utilizado o HSPmédio, utilizado na equação como o tempo de exposição. Mostrado na figura 1. 


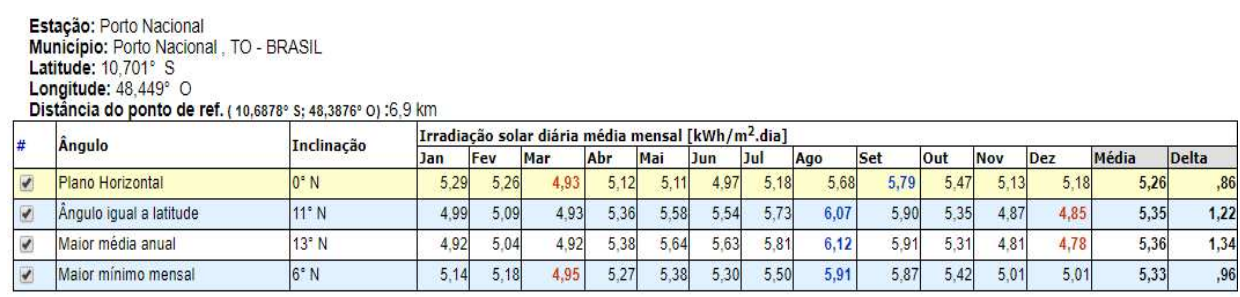

Figura 1: Irradiação solar no plano inclinado, Porto Nacional/TO-Brasil.

A potência pico será calculada pela seguinte equação:

$$
\text { Ppico }=\frac{\text { Ecd }}{\mathrm{HSP}}
$$

Ppico=Potência pico (kWp) $\mathrm{Ecd}=\mathrm{Ecd}=$ Energia de geração por dia $\mathrm{HSP}=$ Horas de sol pico

\section{Número de painéis}

Para calcular a quantidade de painéis foi escolhida dentre as placas comumente utilizadas em residências (260W/265W/275W-1,6X1,0m). Divide-se a potência total de painéis pela potência do painel escolhido.

$$
N m=\frac{\text { Ppico } x 1000}{\mathrm{Wp}}
$$

$\mathrm{Nm}=$ Número de módulos que serão usados no sistema Ppico=Potência de pico do sistema em Kwp $W p=$ Potência de pico do modulo em Wp

\section{Inversor}

Para a escolha do inversor têm-se vários modelos variando suas potencias, ele tem que suprir a potência-pico do sistema, para que faça a conversão das correntes CC em CA. Portanto o valor do inversor pode ser semelhante ao da potência-pico.

\section{Cálculo do Rendimento}

Para completar a equação foi encontrado o rendimento, que são as prováveis perdas que pode acontecer no sistema fotovoltaico, podendo ser por cota da temperatura das placas, incompatibilidade elétrica, acumulo de sujeira, cabeamento, sombreamento o até mesmo pelo próprio inversor, entre outros. Na próxima tabela têm-se os coeficientes de perda e seus valores típicos para cálculo. Para medir essas perdas, são utilizados amperímetros e multímetro.

Tabela 1: Perdas no rendimento.

\begin{tabular}{|l|l|l|l|}
\hline \multicolumn{1}{|c|}{$\begin{array}{c}\text { Coeficiente de } \\
\text { perda por }\end{array}$} & $\begin{array}{c}\text { Simbologia } \\
\text { (padrão } \\
\text { SOLIENS) }\end{array}$ & \multicolumn{1}{c|}{ Descrição } & $\begin{array}{c}\text { Valores } \\
\text { Típicos }\end{array}$ \\
\hline Sombreamento & Ksomb & Causadas por objetos ao redor, ou sobre os módulos fotovoltaicos. & $1 \%$ a $10 \%$ \\
\hline sujeira & Ksuj & $\begin{array}{l}\text { Acúmulo de partículas de poeira, fuligem, fezes de pássaros, etc., sobre os } \\
\text { módulos fotovoltaicos; entre os períodos de limpeza. }\end{array}$ & $2 \%$ a $10 \%$ \\
\hline $\begin{array}{l}\text { Tolerância } \\
\text { Potência }\end{array}$ & Ktol & $\begin{array}{l}\text { Considerada quando a tolerância de potência-pico dos módulos fotovoltaicos } \\
\text { tem algum valor negativo. }\end{array}$ & $1,5 \%$ a 3\% \\
\hline Mismatching & Kmls & $\begin{array}{l}\text { Provocada pelas diferenças de características elétricas entre os módulos } \\
\text { fotovoltaicos. }\end{array}$ & $1 \%$ a $2 \%$ \\
\hline
\end{tabular}




\begin{tabular}{|l|l|l|l|}
\hline $\begin{array}{l}\text { Fator } \\
\text { Temperatura }\end{array}$ & Ktemp & $\begin{array}{l}\text { A potência-pico dos módulos fotovoltaicos diminui com a elevação de sua } \\
\text { temperatura de operação. }\end{array}$ & 5\% 20\% \\
\hline Cabeamento CC & Kcc & Perdas por efeito Joule no círculo CC. & $\begin{array}{l}\text { Perdas durante, ou entre os períodos de rastreamento do ponto de máxima } \\
\text { potência do painel fotovoltaico. }\end{array}$ \\
\hline SPMP & Kspmp a 2\% & $\begin{array}{l}\text { Perdas durante a conversão da energia CC em CA. Não existe inversor com } \\
\text { eficiência 100\% ainda. }\end{array}$ & 1\% a 10\% \\
\hline Conversão CC/CA & Kinv & Perdas por efeito Joule no círculo CA. & 0,5\% a 1\% \\
\hline Cabeamento CA & Kca & & Somatório de perdas, para um sistema típico. \\
\hline & &
\end{tabular}

No caso de sombreamento por árvores poderá pensar na possibilidade de retirada, mas em caso de prédio, ou sombras por causa de obras construídas ao redor, terão que ser previstas essas perdas durante o dia. Com o somatório dessas perdas em porcentagem, elas são subtraídas de $100 \%$ e os resultados são multiplicados pelos fatores de perda, obtendo o rendimento global utilizado na variável.

\section{Cálculo da Potência dos Painéis}

O cálculo da potência total dos painéis é feito substituindo os termos encontrados anteriormente na equação a seguir, assim teremos a potência total. Com esse valor sabe-se qual será a potência mínima necessária para conseguir gerar a energia de geração que aquela local precisa.

$$
P t p=\frac{\mathrm{Eg}}{\mathrm{Te} \times \mathrm{rr}}
$$

Eg- Energia de geração Ptp- Potência total dos painéis Te-Tempo de exposição ao sol Nr-Rendimento

\section{Custos do Sistema}

O custo inicial é o maior custo na equação onde engloba projeto, instalação, custo de equipamentos, contato de distribuidora. Além disso, a manutenção como limpeza, ou substituição de algum componente a partir de 12 anos de uso, caso seja necessário, sendo adicionado de 0,5\% a 1\% do custo inicial ao ano. A taxa da distribuidora também é inserida nesse custo, através do site da ANEEL é encontrada a inflação para base do cálculo. O preço por quilowatt pico é calculado da seguinte forma:

$$
C w p=\frac{\text { Cturn }- \text { key }}{W p}
$$

Cwp=Custo por watt-pico-em $\mathrm{R} \$ / \mathrm{Wp}$

Cturn-key=Custo completo do sistema $W p=$ Potência de pico nominal do sistema

Somando todos os custos envolvidos e dividindo pela geração de energia total, encontra-se o valor do kWh do sistema fotovoltaico, e o foco é que esse valor seja menor que o custo da distribuidora, então:

$$
\text { PreçokWh }=\frac{[\text { Cturn }- \text { key }+ \text { Cinversor }+(\text { Cman x Cturn }- \text { key x 25) }]}{\text { Etotal }}
$$

Cinversor=Custo de substituição do inversor Cman=Custo de manutenção em \% Etotal=Energia total gerada pelo sistema durante sua vida útil kWh

Eanual $(\mathrm{x})=\frac{(\text { Wp x }(1-\text { perdas }) \mathrm{x}[1-(\text { Degrad Módulos x Anoindice })] \mathrm{x} \text { Radiação x 365) }}{1000}$ 
O retorno financeiro é dado da seguinte forma:

$$
\text { RetornoFin }=\text { Eanual }(x) \times\left[\text { Tarifa } x(1+\text { InflaçãoEnerg })^{\text {AnoIndice }}\right]
$$

Retorno Financeiro= Valor gerado pelo sistema FV em R\$

Tarifa $=$ Preço do kWh da distribuidora

Inflação energética= Aumento anual estimado da tarifa de energia da distribuidora, em \%

\section{Cálculo da Fatura de Energia}

A Energisa é responsável pela energia do município de Porto Nacional/TO, essa empresa calcula a conta de energia elétrica com base no seu consumo mensal registrado nas leituras. Com a diferença desses valores é a quantidade de $\mathrm{kW} / \mathrm{h}$ utilizados dentro do mês. Esse valor é multiplicado pelo custo do $\mathrm{kW}$ para obter o valor do seu consumo. Após esse cálculo, são atribuídos os impostos em vigor (PIS, COFINS, ICMS) e a CIP (taxa que custeia a iluminação pública). Em caso de fatura paga com atraso, são cobrados juros, multas e acréscimos moratórios na conta seguinte e, em caso de solicitação de serviços regulados, é cobrada na conta a taxa correspondente a solicitação.

\section{Cálculo do ICMS}

O tipo de alíquota praticado no estado em que se atua faz parte da fatura. No portal do Confaz (Conselho Nacional de Política Fazendária), estão disponíveis os tributos de cada estado, de acordo com a origem e destino da venda. Em uma situação, no qual a venda é efetuada na mesma UF, a fórmula é simples:

\section{Preço do produto $X$ Alíquota praticada no estado = Valor do ICMS da mercadoria}

Exemplo: Um produto custa $\mathrm{R} \$ 80$ reais e sobre ele está uma tarifa de 18\% (valor aplicado no estado do Tocantins), o cálculo seria: $\mathbf{R} \$ \mathbf{8 0} \times \mathbf{2 5 \%}=\mathbf{R} \mathbf{2 0 , 0 0}$

Neste caso o valor do ICMS do produto seria de $\mathrm{R} \$ 20,00$ reais.

\section{Análise de Pis/Pasep e Cofins}

Para o regime cumulativo: Alíquota de 0,65\% para o PIS; e Alíquota 3\% para a COFINS.

Para o regime não cumulativo: Alíquota de PIS é 1,65\%; e Alíquota da COFINS 7,6\%.

\section{Valores das Bandeira Tarifária}

Bandeira verde: não sofre acréscimos.

Bandeira amarela: acréscimo de R\$ $0.010 \mathrm{kWh}$.

Bandeira vermelha: 10 patamar aumenta $\mathrm{R} \$ 0,030 \mathrm{kWh} ; 2$ p patamar aumenta $\mathrm{R} \$ 0,50 \mathrm{kWh}$.

Portanto, para a composição da planilha de dimensionamento do sistema fotovoltaico estão explicitas as metodologias conforme descrito, o cálculo será inserido para uma simulação direta da melhor 
relação entre investimento e retorno financeiro, por isso os tópicos seguintes também são necessários.

\section{Prazo de Retorno do Investimento}

O tempo de retorno do investimento em energia solar, chamado payback, significa o tempo que precisa para o valor de instalação se pague e comece a trazer lucro para o investidor. Para fazer este cálculo foi feito o levantamento do custo total do investimento e dividi-lo pela economia proporcionada mensalmente.

$$
\text { PAYBACK }(\text { meses })=\frac{\text { Investimento }(\mathrm{R} \$)}{\text { Energia gerada }\left(\frac{\mathrm{kWh}}{\mathrm{mes}}\right) \times \text { Valor da Tarifa }}
$$

Esse retorno depende dos fatores como a tarifa da energia elétrica da cidade, o fornecedor contratado e o tamanho do sistema a ser instalado, cada caso precisam ser estudados individualmente, podendo assim variar bastante o payback.

Tabela 2: Fórmulas para cálculo do dimensionamento de sistemas fotovoltaico.

\begin{tabular}{|l|c|}
\hline \multicolumn{1}{|c|}{ Cálculo do dimensionamento de sistemas fotovoltaico } & $\begin{array}{c}E=P \times T \\
\text { Sendo aplicada como } \\
E g=P t p \times T e\end{array}$ \\
\hline Energia & $P t p=\frac{E g}{T e \times N r}$ \\
\hline Potência das placas, com as perdas de energia & Ec $=\mathrm{Cm}-\mathrm{L}$ \\
\hline Cálculo da energia de geração & $E c d=\frac{\mathrm{Dg}}{30}$ \\
\hline Cálculo da energia de geração em dias & Ppico $=\frac{\text { Ecd }}{\mathrm{HSP}}$ \\
\hline Cálculo da potência-pico & Ppico x 1000 \\
\hline Número de painéis & Wp \\
\hline Escolha do Inversor & Deve suprir a potência-pico \\
\hline Cálculo do Rendimento & Encontra-se na Tabela 01 \\
\hline
\end{tabular}

Tabela 3: Custos do sistema e prazo de retorno do investimento.

\begin{tabular}{|l|c|}
\hline $\begin{array}{c}\text { Custos do sistema/Prazo de } \\
\text { retorno }\end{array}$ & EQUAÇõES \\
\hline Preço por quilowatt & Cwp $=\frac{\text { Cturn }- \text { key }}{W p}$ \\
\hline Valor do kWh do sistema & PreçokWh $=\frac{[\text { Cturn }- \text { key }+ \text { Cinversor }+(\text { Cman } \times \text { Cturn }- \text { key } \times 25)]}{\text { Etotal }}$ \\
\hline Energia gerada ao ano & Eanual $(\mathrm{x})=\frac{(\text { Wp x }(1-\text { perdas }) \times[1-(\text { Degrad Módulos } \times \text { Anoindice })] \times \text { Radiação x 365) }}{1000}$ \\
\hline Retorno financeiro & RetornoFin $=$ Eanual $(x) x\left[\right.$ Tarifa $\left.x(1+\text { InflaçãoEnerg })^{\text {AnoIndice }}\right]$ \\
\hline $\begin{array}{l}\text { Prazo de retorno do } \\
\text { investimento }\end{array}$ & PAYBACK(meses $)=\frac{\text { Investimento }(\mathrm{R} \$)}{\text { Energia gerada }\left(\frac{\mathrm{kWh}}{\text { mes }}\right) \times \text { Valor da Tarifa }}$ \\
\hline
\end{tabular}

\section{RESULTADOS E DISCUSSÃO}

A planilha elaborada para analisar a viabilidade e dimensionar os sistemas fotovoltaicos ongrid com intuito de trazer facilidade e agilidade aos projetos dessa categoria, ao engenheiro responsável, foi construída por meio de pesquisas sobre as equações utilizadas para projetar sistemas solares. Com alguns dados dado na fatura do cliente, preços dos materiais/equipamentos para instalação do sistema e informações descritas no site da CRESESB (centro de referência para as energias solar e eólica Sérgio de S. 
Brito), pode-se obter na planilha a energia de geração diária, potência pico, potência total dos painéis, quantidade de painéis necessários, valor total do sistema, o tempo de retorno do investimento, e a diferença entre pagar para a unidade geradora do município ou pelo sistema instalado.

Para melhor entendimento da planilha estarão descritos abaixo o seu procedimento de uso por meio de imagens. No plan1 encontramos primeiro o índice de radiação solar através do site da CRESESB, que difere para cada cidade, obtém todos os valores mensais e sua média será usada para os cálculos. Logo em seguida é encontrada a média do consumo mensal, esses dados são identificados na fatura de energia do cliente. Com o consumo médio e o tipo de ligação, também encontrado na fatura, tem-se a energia de geração por mês e diária, como também a produção média diária. Com a média do índice de radiação solar (Figura 2) e a energia de geração diária (Figura 3), encontra-se a potência pico.

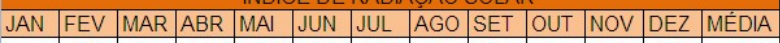

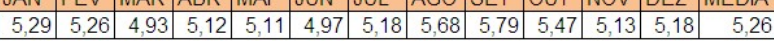

Figura 2: Imagem da planilha, índice de radiação solar.

\begin{tabular}{|l|c|} 
Consumo médio do cliente (CM) & 154,75 \\
\hline Ligação Residencial Monofásica & 30 \\
\hline Energia de geração (Kwhimes) & 124,75 \\
\hline Energia de geração por dia (Kwhidia) & 4,158 \\
\hline Produção média diária & 0 \\
\hline
\end{tabular}

Figura 4: Imagem da planilha, energia de geração diária.

Os coeficientes por perda serão de acordo com os locais onde serão instaladas as placas, para obter essas informações, será necessária uma visita ao local da implantação do sistema, com a determinação desses valores, calcula-se o rendimento total.

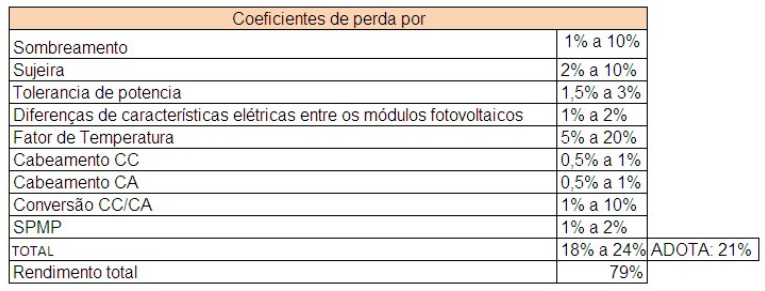

\begin{tabular}{|l|r|}
\hline Energia de geração & 4,158 \\
\hline Tempo de exposição ao sol & 5,26 \\
\hline Rendimento & 0,79 \\
\hline Potencia total dos paineis & 1,001 \\
\hline
\end{tabular}

Figura 7: Imagem da planilha, potência dos painéis.

Figura 6: Imagem da planilha, rendimento total.

\begin{tabular}{|l|r|}
\hline Painel escolhido para residenciais (W) & 260 \\
\hline Potencia Total & 1,001 \\
\hline Quantidade de painéis & 3,849 \\
\hline
\end{tabular}

Figura 8: Imagem da planilha, potência dos painéis.
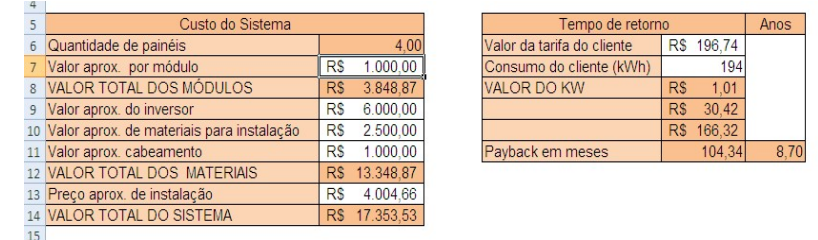

Figura 9: Imagem da planilha, valor total do sistema e tempo de retorno.

Ainda no plan1 da planilha os valores de energia de geração, média do índice de irradiação e rendimento total é dado à potência total dos painéis. Ficará a critério do engenheiro responsável pelo projeto, o tipo de painel escolhido para o sistema, juntamente com a potência total dos painéis é calculada a quantidade de painéis.

No plan2 da planilha com a quantidade de painéis encontrados e os valores dos equipamentos de 
instalação, tem-se o valor total do sistema para a implantação no local que o cliente deseja. Logo após é calculado o tempo de retorno que o sistema levará para definitivamente trazer lucro ao proprietário, em meses e anos, mostrados na Figura 9. A planilha ainda traz ao cliente a diferença entre continuar pagando a energia para a unidade geradora da sua cidade ou pagar pela instalação do sistema fotovoltaico ongrid, o qual, de maneira geral, está exposta na Figura 11.

\section{VALE O INVESTIMENTO?}

Considerando que o cliente pagará durante 105 meses, um valor aproximado, (podendo variar para mais ou para menos) da ultima fatura de energia então:

RS $20.527,96$

Figura 10: Imagem da planilha, investimento ao sistema solar.

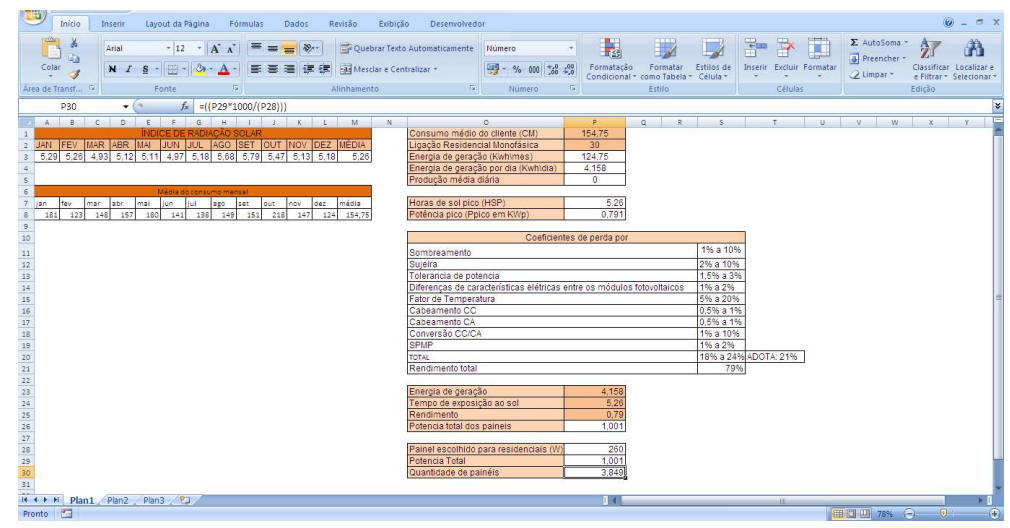

Figura 11: Imagem geral da planilha.

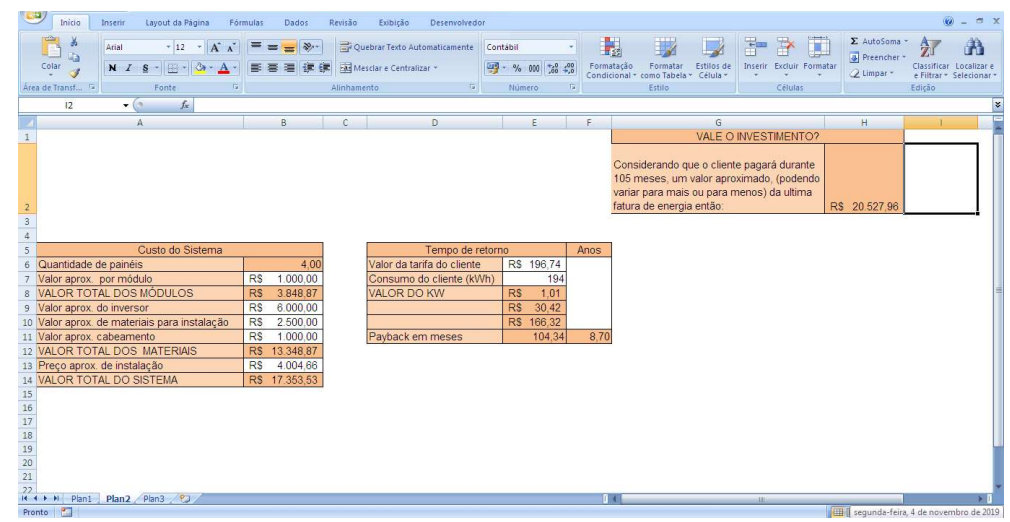

Figura 12: Imagem geral da planilha.

Com essa ferramenta o Engenheiro responsável pelo projeto do sistema terá mais agilidade e facilidade ao calcular os valores do sistema, isso torna a planilha bastante útil para esses profissionais.

\section{CONCLUSÕES}

Conclui-se que o estudo feito para a produção da planilha trouxe resultados significativos para os usuários, como a facilidade do uso dessa ferramenta e a agilidade que ela proporciona ao fazer um projeto de qualquer proporção, por poder ser usada para qualquer tamanho de sistema fotovoltaico, traz conclusões ao cliente e ao engenheiro de antemão sobre os retornos que trará.

A ferramenta só pode ser utilizada mediante a presença de um profissional da área, pois será mais fácil a compreensão do cliente, e os restantes dos passos a serem seguidos para concluir o projeto, que não 
estão presentes na planilha, só poderá ser feito por ele. Assim abrindo portas para novos estudos de construção de outras ferramentas para complementar essa planilha.

Portanto, todo esse estudo foi de grande importância para projetos na área de sistemas fotovoltaicos, trazendo melhoria para esse sistema, e fácil acesso aos profissionais. Espera-se que o estudo continue a se desenvolver trazendo sustentabilidade, prática e facilidade de acesso a todos os públicos, e para todos os tamanhos desse conjunto.

\section{REFERÊNCIAS}

ANEEL. Agência Nacional de Energia Elétrica. Ranking das tarifas. Brasília: ANEEL, 2019.

CEMIG. Companhia Energética de Minas Gerais. Alternativas Energéticas: uma visão Cemig. Belo Horizonte: CEMIG, 2012.

ENERGISA. Como e onde é cobrada a taxa de iluminação pública?. Cataguases: ENERGISA, 1905.

FIGUEIRA, F. F.. Dimensionamento de um Sistema Fotovoltaico Conectado à Rede para Alimentar a Sala de Computação da Escola Municipal Tenente Antônio João.
2014.

IMHOFF, J.. Desenvolvimento de Conversores Estáticos para Sistemas Fotovoltaicos Autônomos. Dissertação (Mestrado em Engenharia Elétrica) - Universidade Federal de Santa Maria, Santa Maria, 2007.

MESSENGER, R.; VENTRE, J.. Photovoltaic Systems Engineering. Boca Raton: CRC Press, 2010.

SOLIENS. Soliens Energia Solar. Belo Horizonte: SOLIENS, 2013.

A CBPC - Companhia Brasileira de Produção Científica (CNPJ: 11.221.422/0001-03) detém os direitos materiais desta publicação. Os direitos referem-se à publicação do trabalho em qualquer parte do mundo, incluindo os direitos às renovações, expansões e disseminações da contribuição, bem como outros direitos subsidiários. Todos os trabalhos publicados eletronicamente poderão posteriormente ser publicados em coletâneas impressas sob coordenação da Sapientiae Publishing, da Companhia Brasileira de Produção Científica e seus parceiros autorizados. Os (as) autores (as) preservam os direitos autorais, mas não têm permissão para a publicação da contribuição em outro meio, impresso ou digital, em português ou em tradução. 